\title{
Capsubot with an opposing spring: Simulation and experiments
}

\author{
A. Nunuparov ${ }^{*}$ F. Becker ${ }^{* *}$ N. Bolotnik ${ }^{* * *}$ I. Zeidis ${ }^{* *}$ \\ K. Zimmermann ${ }^{* *}$ \\ * Moscow Institute of Physics and Technology, 9 Institutskiy per., \\ Dolgoprudny, Moscow Region, 141700, Russia, e-mail: \\ (anunuparov@me.com) \\ ** Technische Universität Ilmenau, Technical Mechanics Group, \\ Germany, e-mail: (felix.becker@tu-ilmenau.de) \\ *** Ishlinsky Institute for Problems in Mechanics RAS, 101-1 \\ Vernadsky Ave, Moscow119526 Russia, (e-mail:bolotnik@ipmnet.ru)
}

Keywords: Mobile robot, Motion control, Capsubot, Locomotion

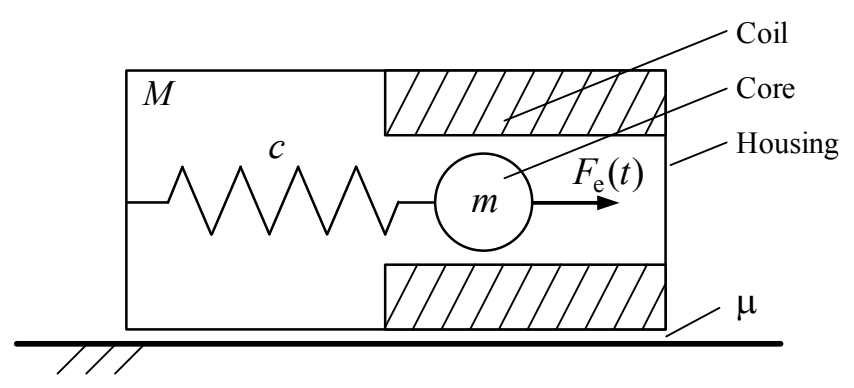

Fig. 1. Capsule-type robot. $M-$ mass of the housing, $m-$ mass of the core, $c$ - spring rate, $F_{e}$ - control force, $\mu$ - coefficient of dry friction

\section{INTRODUCTION}

A capsule robot (capsubot) is a locomotion system that can move in a resistive environment without external propelling devices (legs, wheels, caterpillars, fins, water screws, etc.) due to the motion of internal bodies and interaction of the housing with the environment.

The robot in current study consists of a rigid body (housing) and an electromagnetic (solenoid-type) drive located inside the housing. The drive involves an electromagnetic coil (solenoid) that is rigidly attached to the housing and an internal body(core); the core is made of a ferromagnetic material and can move inside the solenoid along its axis. The core is attached to the housing by a spring. The solenoid's axis is parallel to the axis of the housing. The housing interacts with a resistive environment in which the robot is moving. The robot is actuated by means of a magnetic force that acts on the core when an electric voltage is applied across the solenoid. The drive is designed so that the magnetic force acts in one direction and tends to pull the core inside the coil. The core returns to its initial position due to the spring. The robot moves on a horizontal plane along a straight line parallel to the axis of the robot's housing. The dynamics of the electric circuit

\footnotetext{
* The research work reported here was partly supported by the Deutsche Forschungsgemeinschaft (Grant ZIM 540/19-2) and the Russian Foundation for Basic Research (Grant 17-51-12025)
}

of the solenoid is not taken into account. The schematic of the system described is shown in Fig. 1.

This model was suggested and derived in Bolotnik et al. (2016). The current study extends the cited paper with experimental investigations.

\section{MATHEMATICAL MODEL}

Let $M$ denote the mass of the housing together with the solenoid, $m$ the mass of the core, $F_{\mathrm{e}}$ the force applied to the core by the solenoid, $F_{\text {fr }}$ the force with which the environment resists the motion of the housing, $c$ the spring rate, $x$ the coordinate that identifies the position of the housing's center of mass relative to a fixed (inertial) reference frame, $\xi$ the coordinate that identifies the position of the core's center of mass relative to the housing. The variables $x$ and $\xi$ are measured along the line of motion of the robot. The coordinate $\xi$ is chosen so that the spring is unstrained for $\xi=0$. We assume that the resistance force $F_{\text {fr }}$ acting between the housing and the environment is the dry friction force that obeys Coulomb's law and $\mu$ is the coefficient of dry friction of the housing against the supporting plane. Let $X$ denote the center of mass:

$$
X(t)=x(t)+\frac{m}{M+m} \xi(t)
$$

By applying Newton's second law separately to the housing and to the core and using (1), we obtain the governing equations for the system under consideration in the following form:

$$
\begin{aligned}
& (M+m) \ddot{X}=F_{\text {fr }}\left(\dot{X}-\frac{m}{M+m} \dot{\xi}\right), \\
& \frac{M m}{M+m} \ddot{\xi}+c \xi=F_{\mathrm{e}}-\frac{m}{M+m} F_{\text {fr }}\left(\dot{X}-\frac{m}{M+m} \dot{\xi}\right) .
\end{aligned}
$$

Consider the force generated by the drive as a periodic piecewise continuous function:

$$
F_{\mathrm{e}}=\left\{\begin{array}{l}
F_{0},\left\{\frac{t}{T}\right\}<\tau, \\
0, \quad\left\{\frac{t}{T}\right\} \geq \tau,
\end{array}\right.
$$


where $T$ is the period, $F_{0}$ is a positive constant that has a dimension of force, and $\tau$ is a dimensionless positive constant from the interval $(0,1)$. The parameter $\tau$, called the duty cycle, identifies the fraction of the period, during which the control force is not equal to zero. Curly brackets denote the fractional part of the expression enclosed in them.

\section{SIMULATION AND EXPERIMENTAL RESULTS}

The basic content of this section is the analysis of the dependence of the average velocity of the robot on the excitation parameters $T$ and $\tau$.

The parameters of the experimental setup in terms of the mathematical model are provided in Table 1 . These parameters are used for the simulation.

Table 1. Parameters of the system

\begin{tabular}{lc}
\hline Parameters & Notatation and value \\
\hline Mass of the housing & $M=0.193 \mathrm{~kg}$ \\
Mass of the core & $m=0.074 \mathrm{~kg}$ \\
Stiffness of the spring & $c=256.23 \mathrm{Nm}^{-1}$ \\
Maximum value of the force $F_{e}$ & $F_{0}=1.25 \mathrm{~N}$ \\
Dry friction coefficient & $\mu=0.29$ \\
\hline
\end{tabular}

The results will be presented in dimensionless variables. Instead of $V, t$, and $T$, we will use the variables $V c /\left(F_{0} \omega\right)$, $\omega t$, and $\omega T$, respectively, preserving the previous notation for the normalized variables. The time scaling parameter is defined by $\omega=\sqrt{\frac{c(M+m)}{M m}}$.

In the current study it is expected that the experimental and simulated data will not match quantitatively. This significant discrepancy could be accounted for by the fact that in the computational model, we ignored the dynamics of the electric circuit of the solenoid. However, the qualitative effects are expected to be observed.

\subsection{Dependence of the average velocity of the robot on the parameter $\tau$}

The results of the investigation of the dependence of the average velocity $V$ of the robot on the excitation parameter $\tau$ while the parameter $T$ is fixed are depicted at Figure 2.
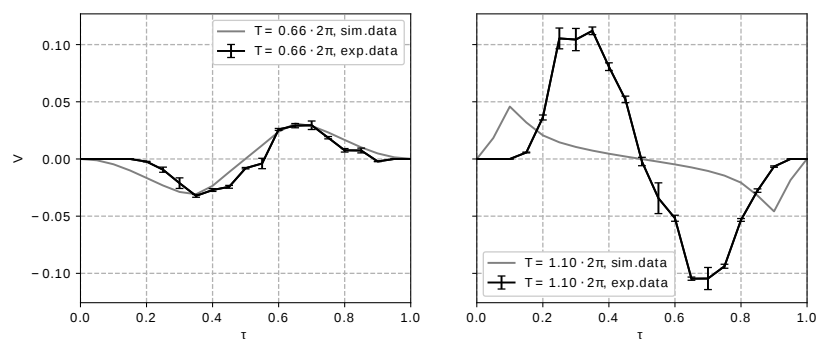

Fig. 2. The dependencies of average velocities on $\tau$

The simulation and the experiment demonstrate that the average velocity of the steady-state motion of the robot depends significantly on the duty cycle of the pulsewidth excitation signal, which indicates the possibility of controlling the motion of the robot by changing only the parameter $\tau$. For $\tau=0$, near $\tau=1 / 2$, and $\tau=1$, the average velocity of the robot is equal to zero. Based on this figure, one can assume that these curves possess a property of central symmetry about the point $(1 / 2,0)$ of the coordinate plane $\tau V$. For the mathematical model used for simulation this property was proved in Bolotnik et al. (2016). This implies that changing the duty cycle of the excitation signal from $\tau$ to $1-\tau$ at the same period leads to the change in the direction of motion of the capsule robot, with the magnitude of its velocity being preserved.

\subsection{Dependence of the average velocity on the period $T$}

Figure 2 shows an essential qualitative difference between the curves for $T=0.66 \cdot 2 \pi$ and $T=1.10 \cdot 2 \pi$, which is reflected in the change in the sign of the average velocities for the same values of $\tau$. For example, the sign of the average velocity in $\tau$ interval from 0 to 0.5 is non-negative for $T=1.10 \cdot 2 \pi>1$ and non-positive for $T=0.66 \cdot 2 \pi<2 \pi$. This effect could be explained by the resonance phenomenon. The resonance-induced change in the direction of motion of a mobile vibration-driven system was observed previously in Zimmermann et al. (2009).

The resonant change in the sign of the average velocity of the robot can be seen from the curve plotting the average velocity $V$ versus the excitation period $T$ (Fig. 3).

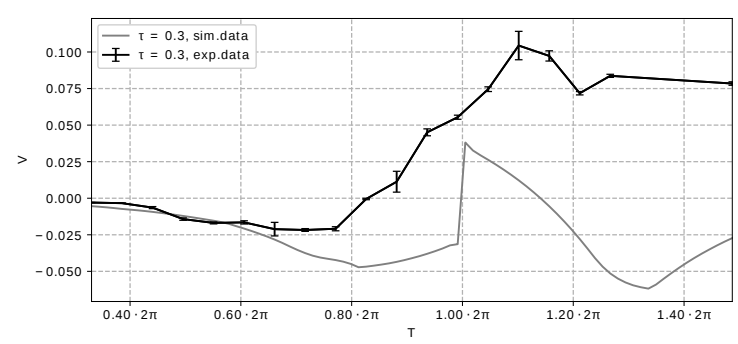

Fig. 3. The dependencies of average velocities on $T$

Both curves, the experiment and the simulation, record the change in the velocity, the first curve near $T=0.82 \cdot 2 \pi$, the second near $T=2 \pi$. Thus, the resonance effect is observed in the experiment and in the simulation.

\section{CONCLUSION}

This paper provides model-based and experimental investigations of a capsule-type robot motion with a periodic excitation force. The excitation force was controlled by two excitation parameters. It was demonstrated that the magnitude and the sign of the average velocity can be controlled by tuning any of the excitation parameters. The resonance-induced change in the direction of motion is shown.

\section{REFERENCES}

Bolotnik, N., Nunuparov, A., and Chashchukhin, V. (2016). Capsule-type vibration-driven robot with an electromagnetic actuator and an opposing spring: Dynamics and control of motion. Journal of Computer and Systems Sciences International, 55, 986-1000.

Zimmermann, K., Zeidis, I., Bolotnik, N., and Pivovarov, M. (2009). Dynamics of a two-module vibration-driven system moving along a rough horizontal plane. Multibody System Dynamics, 22, 199-219. 